Directeur de LA PUblication / Publication diRECTOR: Bruno David,

Président du Muséum national d'Histoire naturelle

RÉDACTEUR EN CHEF / EDITOR-IN-CHIEF: Jean-Philippe Siblet

ASSISTANTE DE RÉDACTION / ASSISTANT EDITOR: Sarah Figuet (naturae@mnhn.fr)

Mise EN PAGE / PAGE LAYOUT: Sarah Figuet

COMITÉ SCIENTIFIQUE / SCIENTIFIC BOARD:

Luc Abbadie (UPMC, Paris)

Luc Barbier (Parc naturel régional des caps et marais d'Opale, Colembert)

Aurélien Besnard (CEFE, Montpellier)

Vincent Boullet (Expert indépendant flore/végétation, Frugières-le-Pin)

Hervé Brustel (École d'ingénieurs de Purpan, Toulouse)

Patrick De Wever (MNHN, Paris)

Thierry Dutoit (UMR CNRS IMBE, Avignon)

Éric Feunteun (MNHN, Dinard)

Romain Garrouste (MNHN, Paris)

Grégoire Gautier (DRAAF Occitanie, Toulouse)

Olivier Gilg (Réserves naturelles de France, Dijon)

Frédéric Gosselin (Irstea, Nogent-sur-Vernisson)

Patrick Haffner (UMS PatriNat, Paris)

Frédéric Hendoux (MNHN, Paris)

Xavier Houard (OPIE, Guyancourt)

Isabelle Leviol (MNHN, Concarneau)

Francis Meunier (Conservatoire d'espaces naturels - Hauts-de-France, Amiens)

Serge Muller (MNHN, Paris)

Francis Olivereau (DREAL Centre, Orléans)

Laurent Poncet (UMS PatriNat, Paris)

Nicolas Poulet (OFB, Vincennes)

Jean-Philippe Siblet (UMS PatriNat, Paris)

Laurent Tillon (ONF, Paris)

Julien Touroult (UMS PatriNat, Paris)

COUVERTURE / COVER:

Ripisylve à forte capacité d'accueil pour la biodiversité riveraine. Crédit photo: Sébastien De Danieli, 2019, INRAE.

Naturae est une revue en flux continu publiée par les Publications scientifiques du Muséum, Paris

Naturae is a fast track journal published by the Museum Science Press, Paris

Les Publications scientifiques du Muséum publient aussi / The Museum Science Press also publish:

Adansonia, Zoosystema, Anthropozoologica, European Journal of Taxonomy, Geodiversitas, Cryptogamie sous-sections Algologie, Bryologie, Mycologie, Comptes Rendus Palevol.

Diffusion - Publications scientifiques Muséum national d'Histoire naturelle

CP $41-57$ rue Cuvier F-75231 Paris cedex 05 (France)

Tél. : 33 (0)1 40794805 / Fax: 33 (0)1 40793840

diff.pub@mnhn.fr / https://sciencepress.mnhn.fr

(c) Publications scientifiques du Muséum national d'Histoire naturelle, Paris, 2021

ISSN (électronique / electronic): 1638-9387 


\title{
Évaluer la qualité des boisements riverains avec l'Indice de Biodiversité et de Connectivité des Ripisylves (IBCR): une étude de cas avec les communautés d'oiseaux
}

\author{
Philippe JANSSEN \\ André EVETTE \\ Laurent BERGÈS \\ Université Grenoble Alpes, INRAE, \\ 2 rue de la Papeterie boîte postale 76, F-38402 Saint-Martin-d'Hères (France) \\ philippe.janssen@inrae.fr \\ andre.evette@inrae.fr \\ laurent.berges@inrae.fr
}

Pierre GONIN

Institut pour le Développement forestier (IDF), Maison de la Forêt,

7 chemin de la Lacade, F-31320 Auzeville Tolosane (France) pierre.gonin@cnppf.fr

Laurent LARRIEU

Université de Toulouse, INRAE,

24 Chemin de Borde Rouge, F-31320 Castanet-Tolosan (France) et Centre national de la Propriété forestière (CNPF) - Centre régional de la Propriété forestière (CRPF) Occitanie, Occitanie, Maison de la Forêt,

7 chemin de la Lacade, F-31320 Auzeville Tolosane (France) laurent.larrieu@inrae.fr

Mélanie DAJOUX Stéphanie DUPONT Stéphane GARDIEN Christophe GILLES Alain LADET

France Nature Environnement Auvergne-Rhône-Alpes, HEVEA-Etic,

2 rue professeur Zimmermann, F-69007 Lyon (France) melanie.dajoux@fne-aura.org stephanie.dupont@fne-aura.org stephane.gardien@fne-aura.org christophe.gilles@fne-aura.org alain.ladet@wanadoo.fr

Soumis le 20 novembre 2020 | Accepté le 29 janvier 2021 | Publié le 6 octobre 2021

Janssen P., Evette A., Bergès L., Gonin P., Larrieu L., Dajoux M., Dupont S, Gardien S., Gilles C. \& Ladet A. 2021. Évaluer la qualité des boisements riverains avec l'Indice de Biodiversité et de Connectivité des Ripisylves (IBCR): une étude de cas avec les communautés d'oiseaux. Naturae 2021 (21): 293-307. https://doi.org/10.5852/naturae2021a21

RÉSUMÉ

Afin d'évaluer la capacité d'accueil des ripisylves pour la biodiversité, nous proposons un nouvel indice tenant compte des dimensions longitudinale et transversale de l'hydrosystème: l'Indice de Biodiversité et de Connectivité des Ripisylves (IBCR). Constitué de 15 facteurs renseignant des critères liés au peuplement et à sa gestion, au contexte environnant, aux perturbations/pressions 
MOTS CLÉS Avifaune, diagnostic écologique, indicateur indirect, milieux riverains.

KEY WORDS Avifauna, ecological diagnosis, indirect indicators, riparian environments. et à la connectivité, l'IBCR est un nouvel outil qui fournit aux gestionnaires des cours d'eau des éléments concrets pour diagnostiquer rapidement l'état écologique des ripisylves et orienter la gestion pour la conservation de la biodiversité associée aux linéaires boisés riverains. Pour illustrer l'utilité de cet indice, nous avons étudié la réponse des communautés d'oiseaux aux facteurs de l'IBCR, en distinguant les espèces des milieux forestiers, agricoles, aquatiques et bâtis/rocheux. Nos résultats montrent que les cortèges d'oiseaux répondent de façon assez cohérente aux valeurs de l'IBCR, avec une augmentation de la richesse en espèces d'oiseaux des milieux forestiers avec l'augmentation du score moyen de l'indice. En revanche, les ripisylves ayant un indice de faible score, c'est-à-dire davantage dégradées et déconnectées, favorisent surtout la présence d'espèces d'oiseaux des milieux agricoles. Dans l'ensemble, ces résultats soulignent l'intérêt de l'IBCR pour évaluer rapidement les principales caractéristiques des boisements riverains et les capacités d'accueil associées, au moins pour les communautés d'oiseaux.

\begin{abstract}
Assessing the quality of riparian woodlands with the Index of Biodiversity and Connectivity of Riparian forest stands (IBCR): a case study with bird communities.

In order to assess the carrying capacity of riparian forest stands for biodiversity, we propose a new index that takes into account the longitudinal and cross-sectional dimensions of the hydrosystem: the Index of Biodiversity and Connectivity for Riparian forests (IBCR). Consisting of 15 factors that provide information on criteria related to the stand attributs and its management, the surrounding context, disturbance/pressure and connectivity, the IBCR is a new tool that provides river managers with concrete elements to quickly diagnose the ecological status of riparian forest stands and guide management for the conservation of the biodiversity associated with these linear forests. To illustrate the usefulness of this index, we have studied the response of bird communities to IBCR factors, distinguishing between species in forest, agricultural, aquatic and built/rocky environments. Our results show that bird communities respond fairly consistently to the values of the IBCR forests, with an increase in the species richness of forest birds with the mean score of the index. On the other hand, the riparian forest stands with a low score index, i.e. more degraded and disconnected, favour above all the presence of agricultural bird species. Overall, these results underline the interest of the IBCR to rapidly assess the main characteristics of riparian forest stands and the associated carrying capacities, at least for bird communities.
\end{abstract}

\section{INTRODUCTION}

Les ripisylves constituent un espace de transition entre le cours d'eau et le milieu terrestre. Ces linéaires boisés, plus ou moins denses et plus ou moins larges selon les habitats naturels et la topographie des lieux, forment ainsi un écotone constitué de peuplements forestiers caractéristiques sous la dépendance d'un régime de perturbations spécifique (inondations, phénomènes d'érosion-dépôt) et souvent d'une nappe d'accompagnement (Dufour \& Piégay 2006). Du fait de cette position à l'interface entre écosystèmes terrestre et aquatique, les ripisylves sont reconnues pour la multitude des fonctions (régulation des débits, stabilisation des berges) et des services (contrôle des inondations, qualité de l'eau) qu'elles assurent, et participent in fine au bon état écologique du cours d'eau (Naiman \& Decamps 1997). Plus généralement, les formations boisées riveraines sont considérées comme des éléments naturels jouant un rôle écologique majeur comparativement à leur taille restreinte. En effet, bien qu'à peine $1 \%$ de la surface continentale européenne soit occupée par les forêts riveraines (Weissteiner et al. 2016), ces milieux accueillent un cortège d'espèces unique et sont d'une importance critique pour la conservation de la biodiversité (Gregory et al. 1991; Naiman \& Decamps 1997).
Àl'échelle mondiale, les hydrosystèmes sont parmi les écosystèmes les plus impactés par les activités humaines (Nilsson et al. 2005) et probablement parmi les plus vulnérables aux changements globaux en cours (Perry et al. 2012). Pour contrôler le risque d'inondation et l'érosion des berges, des techniques lourdes de génie civil (e.g., endiguements, barrages) ont été massivement utilisées, altérant les dynamiques hydro-géomorphologiques et écologiques des hydrosystèmes, avec de graves conséquences pour la biodiversité riveraine (Dudgeon et al. 2006; Poff et al. 2007; Tonkin et al. 2018). Associées à diverses pressions d'origine anthropique (changement d'usage des sols, pollution, pompage, etc.), ces altérations conduisent à la banalisation, voire à la destruction des communautés riveraines. De nombreuses études ont ainsi montré que la canalisation des rivières et la régulation des débits favorisaient les espèces exotiques envahissantes en forêts alluviales, réduisaient la diversité des essences autochtones et homogénéisaient la structure des peuplements forestiers (Oswalt \& King 2005; Dufour et al. 2007; Janssen et al. 2020). Face à ces dégradations et afin d'améliorer la qualité de l'eau et des habitats riverains, comme voulu par la Directive-cadre européenne sur l'eau de 2000, l'évaluation de l'état écologique des zones riveraines est devenu un enjeu majeur et un préalable à la conduite d'opérations de restauration. 
La complexité des milieux naturels, notamment des milieux d'interface comme les zones riveraines, et la multitude des paramètres qui influencent la biodiversité, rendent l'étude de ces écosystèmes très difficile et chronophage. Pour pallier ces limites et fournir des outils pratiques aux gestionnaires, des indicateurs indirects de l'état écologique des habitats riverains ont été proposés (Munné et al. 2003; Meixler \& Bain 2010 ; González del Tánago \& García de Jalón 2011). Cependant, ces indicateurs sont souvent calibrés à l'échelle d'un tronçon de rivière, dépassant généralement l'emprise des ripisylves, et ne prennent pas en compte des attributs spécifiques des peuplements forestiers comme les bois morts et les dendromicrohabitats, pourtant cruciaux pour bon nombre d'espèces forestières (Stokland et al. 2012). À l'inverse, pour les écosystèmes forestiers non rivulaires, des indicateurs indirects de biodiversité à l'échelle du peuplement intégrant des liens fonctionnels entre attributs des peuplements et biodiversité ont été développés, comme par exemple l'Indice de Biodiversité potentielle (IBP, Larrieu \& Gonin 2008). Bien que calibré pour un vaste ensemble climatique et une large diversité de peuplements forestiers (Larrieu et al. 2012), l'IBP ne prend pas en compte plusieurs facteurs biotiques (espèces exotiques envahissantes), abiotiques et anthropiques (artificialisation, dérangement et perturbation), fonctionnels (connectivité) a priori déterminants pour évaluer la capacité d'accueil des ripisylves pour la biodiversité.

Dans cet article, nous présentons un nouvel indice, l'Indice de Biodiversité et de Connectivité des Ripisylves (IBCR), développé pour évaluer la qualité des ripisylves pour la diversité des espèces vivant dans ces linéaires boisés. Basé sur l'IBP, cet indice s'en distingue par l'ajout de facteurs prenant en compte les dimensions longitudinales et transversales de l'hydrosystème pour l'évaluation globale et rapide des principales caractéristiques des boisements riverains. Afin de calibrer l'indice avec des données empiriques de biodiversité, nous avons testé l'influence du score de l'IBCR et de chacun des facteurs qui le constituent, sur la richesse et la composition des communautés d'oiseaux. Le choix des oiseaux comme modèle d'étude répond à des considérations :

- écologiques, liées à des exigences d'habitat contrastées, à la fois locales et paysagères, mais aussi à une sensibilité démontrée à la qualité des ripisylves (Archaux \& Martin 2009);

- scientifiques, liées à l'utilisation de ce même groupe taxonomique pour calibrer l'IBP (Larrieu et al. 2019), rendant ainsi les comparaisons entre IBCR et IBP pertinentes;

- pratiques, liées à la disponibilité de compétences locales pour réaliser les inventaires, tout en présentant un bon ratio pertinence/coût (Larrieu et al. 2018).

Cette approche visant à étudier la relation entre des mesures de biodiversité réelle et des indicateurs indirects de biodiversité reste encore marginale, notamment pour des métriques autres que la richesse spécifique (Gao et al. 2015). Dans ce cadre, l'objectif de cet article est d'une part de porter à connaissance le nouvel indice développé et, d'autre part, de justifier l'intérêt de son utilisation par les gestionnaires des milieux riverains pour évaluer la qualité des ripisylves à partir de quelques facteurs mesurés sur le terrain.

\section{MATÉRIEL \& MÉTHODE}

\section{L'INDICE DE BIODIVERSITÉ \\ ET DE CONNECTIVITÉ DES RIPISYLVES (IBCR)}

L'IBCR se compose de 15 facteurs, pour lesquels de nombreux liens ont été établis dans la littérature scientifique avec des métriques de biodiversité (Tableau 1). L'utilisateur de l'IBCR attribue un score allant de 0 à 5 (avec trois ou quatre modalités possibles) à chacun de ces facteurs en fonction de seuils prédéfinis (pour plus de détails, le lecteur pourra se reporter à la fiche et la notice explicative détaillée de l'IBCR disponible en ligne, https ://www.fne-aura.org/ripisylves, dernière consultation le 10 avril 2020). En mode plafonné (i.e. le mode utilisé en routine par le gestionnaire), l'observateur, lors du parcours, arrête de renseigner un facteur dès que le score maximal est atteint pour ce facteur (par exemple, pour le facteur C, lorsqu'il a compté huit items de gros bois mort debout, ce qui confere au facteur le score de 5) et ne se concentre alors que sur les facteurs qui n'ont pas encore atteint le score maximal. En faisant cela, il réduit l'effort d'observation et le temps de notation. Ordonnée en quatre grands ensembles, la prise en compte des 15 facteurs permet d'attribuer un score total à la ripisylve variant entre 0 pour les ripisylves à la fois très dégradées (par exemple une peupleraie gérée intensivement) et très peu connectées, et 100 pour les ripisylves à la fois de très bonne qualité (par exemple une forêt galerie de Saules blancs Salix alba L. riche en très gros arbres et en bois mort) et très bien connectées à l'hydrosystème. Il s'agit plus spécifiquement de renseigner:

- sept facteurs liés au peuplement et à la gestion, incluant six facteurs issus de l'IBP et adaptés au contexte spécifique des ripisylves: présence de ligneux autochtones caractéristiques des ripisylves (facteur A), en faisant la distinction entre les essences à bois tendre et à bois dur, structure verticale de la végétation (B), nombre de pièces de bois mort au sol (C) et sur pied (D), de très gros bois vivants $(\mathrm{E})$, d'arbres porteurs de dendromicrohabitats $(F)$ et d'abris racinaires aquatiques $(G)$;

- trois facteurs liés au contexte, issus de l'IBP et adaptés aux ripisylves : continuité temporelle de l'état boisé $(\mathrm{H})$, présence de milieux aquatiques complémentaires au cours d'eau (I) et de milieux minéraux et annexes (par exemple une plage de dépôts de sédiments fins) ( $\mathrm{J}$ ) ;

- deux facteurs liés aux perturbations/pressions sur la ripisylve, propres à l'IBCR: présence d'arbres exotiques et d'espèces invasives $(\mathrm{K})$ et état de dégradation (par exemple enrochement des pieds de berge) et de perturbation (par exemple surfréquentation) du milieu (L), du fait des activités anthropiques;

- trois facteurs liés à la connectivité de la ripisylve, propres à l'IBCR: connectivité longitudinale (c'est-à-dire la continuité du couvert arboré le long du cours d'eau) (M), transversale (c'est-à-dire les liens avec le lit majeur) $(\mathrm{N})$ et paysagère (c'està-dire le contexte environnant) $(\mathrm{O})$ de la ripisylve.

Le domaine d'application de l'IBCR se limite a priori aux ripisylves des cours d'eau du bassin Rhône Méditerranée (excluant le pourtour méditerranéen, pour lequel des spécificités existent en termes d'essences et d'attributs des maturités). Il s'applique aux berges directement en contact 
TABLEAU 1. - Présentation des 15 facteurs constituant l'Indice de Biodiversité et de Connectivité des Ripisylves (IBCR) : définitions, scores associés et liens avec l'Indice de Biodiversité potentielle (IBP) (voir aussi en ligne la fiche et la notice explicative: https://www.fne-aura.org/ripisylves/boite-a-outils, dernière consultation le 10 avril 2020).

\begin{tabular}{|c|c|c|c|c|}
\hline \multicolumn{2}{|c|}{ Facteurs de I'IBCR } & Définition & Score & IBP \\
\hline $\begin{array}{l}\text { Facteurs liés au } \\
\text { peuplement } \\
\text { et à la gestion }\end{array}$ & $\begin{array}{l}\text { A - Ligneux autochtones } \\
\text { caractéristiques } \\
\text { B - Structure verticale de la } \\
\text { végétation } \\
\text { C - Bois morts sur pied } \\
\text { D - Bois morts au sol } \\
\text { E - Très gros bois vivants } \\
\text { F - Arbres vivants porteurs } \\
\text { de dendro-microhabitats } \\
\text { G - Abris racinaires } \\
\text { aquatiques }\end{array}$ & $\begin{array}{l}\text { Nombre de groupements ligneux à bois tendre } \\
\text { et à bois dur } \\
\text { Nombre de strates de végétation } \\
\text { Nombre de bois morts sur pied } \\
\text { Nombre de bois et de tas de bois morts au sol } \\
\text { Nombre d'arbres vivants de gros diamètre } \\
\text { Nombre d'arbres vivants porteurs de dendro-microhabitats } \\
\text { Nombre d'entrelacs racinaires et de cavités formées } \\
\text { par les contreforts racinaires }\end{array}$ & $\begin{array}{l}0-2-5 \\
0-2-5 \\
0-1-2-5 \\
0-1-2-5 \\
0-1-2-5 \\
0-2-5 \\
0-2-5\end{array}$ & $\begin{array}{l}\text { Oui } \\
\text { Oui } \\
\text { Oui } \\
\text { Oui } \\
\text { Oui } \\
\text { Oui } \\
\text { Non }\end{array}$ \\
\hline $\begin{array}{l}\text { Facteurs liés au } \\
\text { contexte }\end{array}$ & $\begin{array}{l}\text { H - Continuité temporelle de } \\
\text { l'état boisé } \\
\text { I - Milieux aquatiques } \\
\text { complémentaires au cours } \\
\text { d'eau } \\
\text { J - Milieux minéraux } \\
\text { et annexes }\end{array}$ & $\begin{array}{l}\text { Présence continue d'un couvert forestier attesté } \\
\text { sur les documents d'archive (carte d'état-major) } \\
\text { Nombre de milieux aquatiques complémentaires d'origine } \\
\text { naturelle ou artificielle } \\
\text { Nombre de milieux minéraux et annexes }\end{array}$ & $\begin{array}{l}0-2-5 \\
0-2-5\end{array}$ & $\begin{array}{l}\text { Oui } \\
\text { Oui }\end{array}$ \\
\hline $\begin{array}{l}\text { Facteurs liés aux } \\
\text { perturbations du } \\
\text { milieu }\end{array}$ & $\begin{array}{l}\text { K - Présence d'arbres } \\
\text { exotiques et d'espèces } \\
\text { invasives } \\
\text { L - Dégradations et } \\
\text { perturbations du milieu }\end{array}$ & $\begin{array}{l}\text { Pourcentage de recouvrement des espèces exotiques } \\
\text { et/ou invasives } \\
\text { Nombre de perturbations d'origine anthropique } \\
\text { (palplanches, sur-fréquentation, décharges, etc.) }\end{array}$ & $0-2-4-5$ & Non \\
\hline $\begin{array}{l}\text { Facteurs de } \\
\text { connectivité }\end{array}$ & $\begin{array}{l}\text { M - Connectivité longitudinale } \\
\text { N - Connectivité transversale } \\
\text { O - Connectivité paysagère }\end{array}$ & $\begin{array}{l}\text { Pourcentage de couvert arboré sur la berge } \\
\text { Largeur des ouvrages coupant le cours d'eau } \\
\text { Degré de connexion de la berge au lit majeur } \\
\text { Pourcentage de sol nu } \\
\text { Pente moyenne de la berge } \\
\text { Distance aux boisements environnants } \\
\text { Nombre de routes environnantes } \\
\text { Nombre de bâtiments environnants }\end{array}$ & $\begin{array}{l}0-2-4-5 \\
0-2-4-5 \\
0-2-4-5 \\
0-2-4-5 \\
0-2-4-5 \\
0-2-4-5 \\
0-2-4-5 \\
0-2-4-5\end{array}$ & $\begin{array}{l}\text { Non } \\
\text { Non } \\
\text { Non } \\
\text { Non } \\
\text { Non } \\
\text { Non } \\
\text { Non } \\
\text { Non }\end{array}$ \\
\hline
\end{tabular}

avec le cours d'eau et souvent de faible largeur (quelques mètres à une dizaine de mètres). Au-delà de ce linéaire boisé, comme par exemple dans les vastes ensembles de forêts alluviales, l'IBP est mieux adapté pour évaluer la capacité d'accueil du peuplement forestier en espèces, ce qui n'exclut pas pour autant la prise en compte de facteurs complémentaires, comme par exemple la présence de certaines espèces exotiques, si l'on souhaite évaluer l'état de conservation de l'habitat (Larrieu et al. 2012).

\section{ZONE D'ÉTUDE}

La zone d'étude est localisée au niveau des piémonts des Alpes du Nord et du Jura, dans les départements de l'Ain, de la Savoie et de la Haute-Savoie (Fig. 1) et se caractérise par un substratum à dominante calcaire et un climat montagnard tempéré. Afin de restreindre la zone d'étude à un contexte écologique le plus homogène possible et de contrôler au maximum les variations dans les paramètres confondants (notamment climatiques), une succession de filtres cartographiques a été appliquée via un système d'information géographique géré sous Qgis (Quantum QGIS Development Team 2016). Pour cela, seuls les cours d'eau permanents, d'ordre 3 à 4 (au sens de la classification de Strahler), situés entre $200 \mathrm{~m}$ et $500 \mathrm{~m}$ d'altitude, ont été sélectionnés. Aussi, afin de cibler un contexte paysager homogène et d'assurer une plus grande indépendance des observations, un maillage systématique de $5 \times 5 \mathrm{~km}$ a été appliqué sur les linéaires de cours d'eau sélectionnés, en écartant les mailles incluant des zones fortement artificialisées (surface bâtie $>3 \%$ ) et/ou fortement dominées par la forêt (surface boisée $>70 \%$ ). Au final, le plan d'échantillonnage est constitué de 53 ripisylves des petits et moyens cours d'eau permanents de piémont incluses dans un contexte paysager à dominante agricole (Figs 1,2).

\section{RELEVÉS D'IBCR}

Au sein de chacune des 53 mailles de $5 \times 5 \mathrm{~km}$ sélectionnées, un tronçon continu et homogène (structure et composition) de $500 \mathrm{~m}$ de ripisylve a été recherché et échantillonné. Pour chaque tronçon, deux relevés, un sur chaque rive, ont été effectués à l'aide de l'Indice de Biodiversité et de Connectivité des Ripisylves (IBCR) en renseignant une fiche dédiée. Pour chaque rive, un même observateur a parcouru le linéaire boisé en renseignant directement sur le terrain les facteurs liés au peuplement et à la gestion (A-G), une partie des facteurs liés au contexte (I, J) et les facteurs liés aux perturbations du milieu (K, L). Afin de pouvoir confronter chacun des facteurs avec les données taxonomiques en disposant du plus large gradient de valeurs possible, les relevés ont été effectués en mode "déplafonné», c'est-à-dire sans stopper la notation du facteur lorsque les observations atteignent le score maximal 


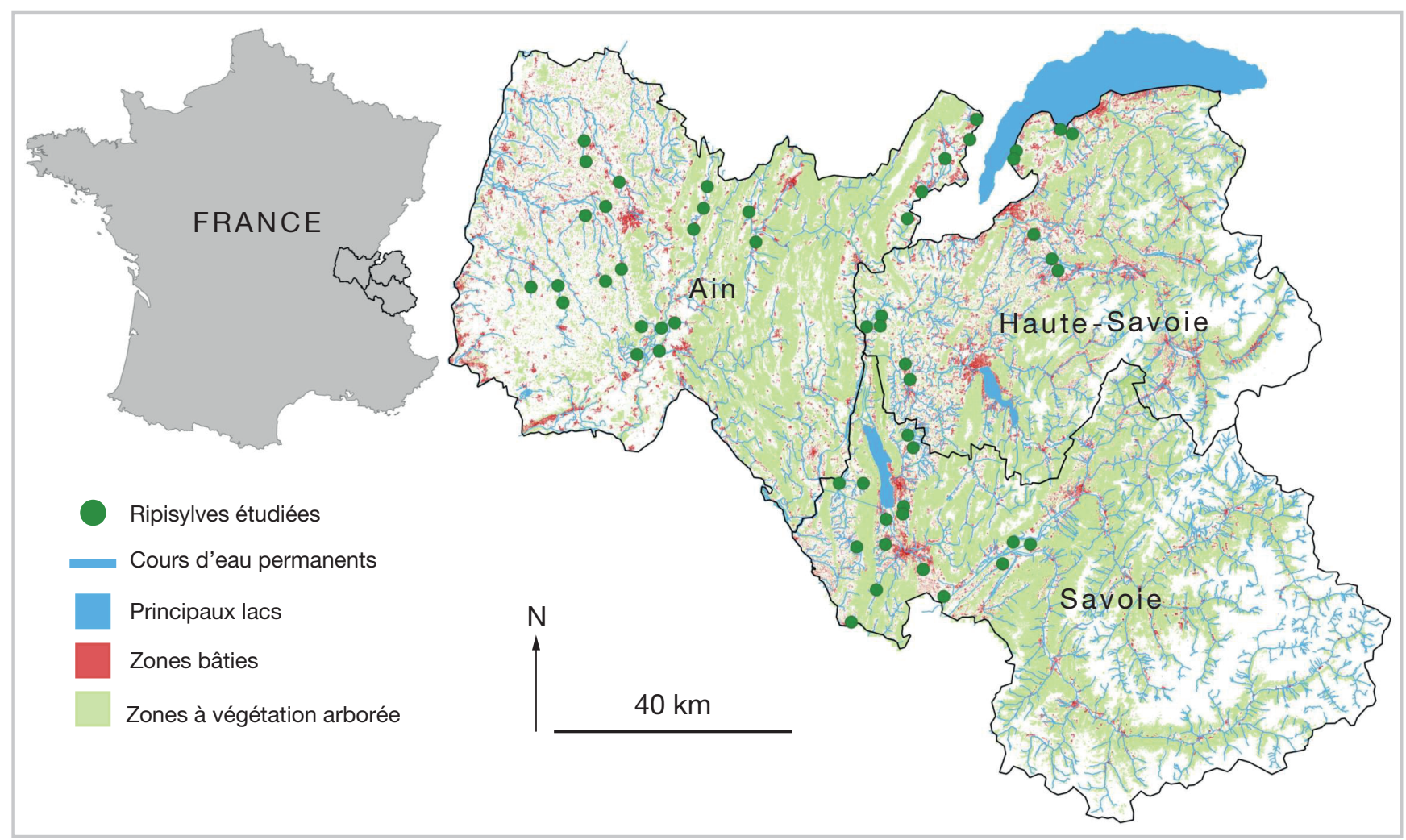

FIG. 1. - Localisation de la zone d'étude en France et répartition des ripisylves étudiées dans les piémonts des Alpes du Nord et du Jura.

du facteur. Les autres facteurs, notamment de connectivités, ont été renseignés au bureau en mobilisant d'une part des documents d'archives $(\mathrm{H})$ et, d'autre part, des orthophotographies récentes des zones étudiées $(\mathrm{M}, \mathrm{N}, \mathrm{O})$.

\section{RELEVÉS DE BIODIVERSITÉ RÉELLE}

Pour chaque ripisylve, la méthode de l'Indice ponctuel d'Abondance (IPA) a été utilisée pour caractériser les communautés d'oiseaux (Blondel et al. 1970). Deux relevés standardisés et reproductibles visent à inventorier l'ensemble des espèces d'oiseaux vues ou entendues, quelle que soit la distance entre l'oiseau et l'observateur, pendant une période de 20 min par un même observateur immobile, localisé au centre de la zone de relevé de la ripisylve. Cette approche standardisée présente l'avantage de faire le focus sur les cortèges d'oiseaux nicheurs ou susceptibles de l'être en ripisylve, mais l'inconvénient d'inclure des espèces détectables au-delà des limites de la ripisylve ou seulement de passage. Un premier relevé a été réalisé entre la mi-avril et la mi-mai 2019 pour inventorier les oiseaux nicheurs précoces et un second relevé, sur les mêmes points d'écoute, entre la mi-mai et la mi-juin 2019 pour inventorier les oiseaux nicheurs tardifs (voir la liste complète des espèces inventoriées en Annexe 1). Chaque IPA a été réalisé entre 6h00 et $9 \mathrm{~h} 00 \mathrm{du}$ matin, au moment de la journée où les oiseaux sont les plus actifs, et dans de bonnes conditions climatiques, c'est-à-dire sans pluie ni vent.

Afin de tenir comptes des exigences d'habitat différentes entre les espèces mais aussi de limiter les biais associés à la méthode d'inventaire utilisée (espèces de passage et/ou associées à des habitats connexes), la réponse des cortèges d'oiseaux à l'IBCR et aux facteurs le composant a été analysée en distinguant les espèces dépendantes a priori des milieux forestiers (par exemple: Mésange bleue, Pic noir, Sittelle torchepot), agricoles (par exemple: Bruant zizi, Fauvette grisette, Pie-grièche écorcheur), aquatiques (par exemple: Canard colvert, Héron cendré, Martin-pêcheur d'Europe) et bâtis ou rocheux (par exemple: Bergeronnette grise, Moineau domestique, Tourterelle turque). Cette classification repose sur une typologie élaborée à dire d'experts et renseignant pour chaque espèce d'oiseau nicheur de France son habitat principal, en considérant trois niveaux hiérarchiques emboités: domaine (cinq classes), paysage (11 classes) et milieu (35 classes) (Roché et al. 2016).

\section{ANALYSES STATISTIQUES}

Pour chaque site $(n=53)$, les IBCR réalisés en rives droite et gauche ont été combinés en calculant la moyenne des deux scores, pour l'indice ainsi que pour chaque facteur individuel.

Dans un premier temps, les analyses ont porté sur les variations de diversité en considérant d'une part les valeurs de la note IPA et d'autre part la richesse en espèces d'oiseaux des milieux forestiers, agricoles, aquatiques et bâtis/rocheux. Pour chacune de ces variables à expliquer, nous avons comparé 17 modèles statistiques, testant le pouvoir prédictif du score moyen de l'IBCR et du score moyen de chacun des facteurs individuels $(\mathrm{n}=15)$, plus un modèle nul. Nous avons utilisé un modèle de distribution normal (LM) pour relier la 


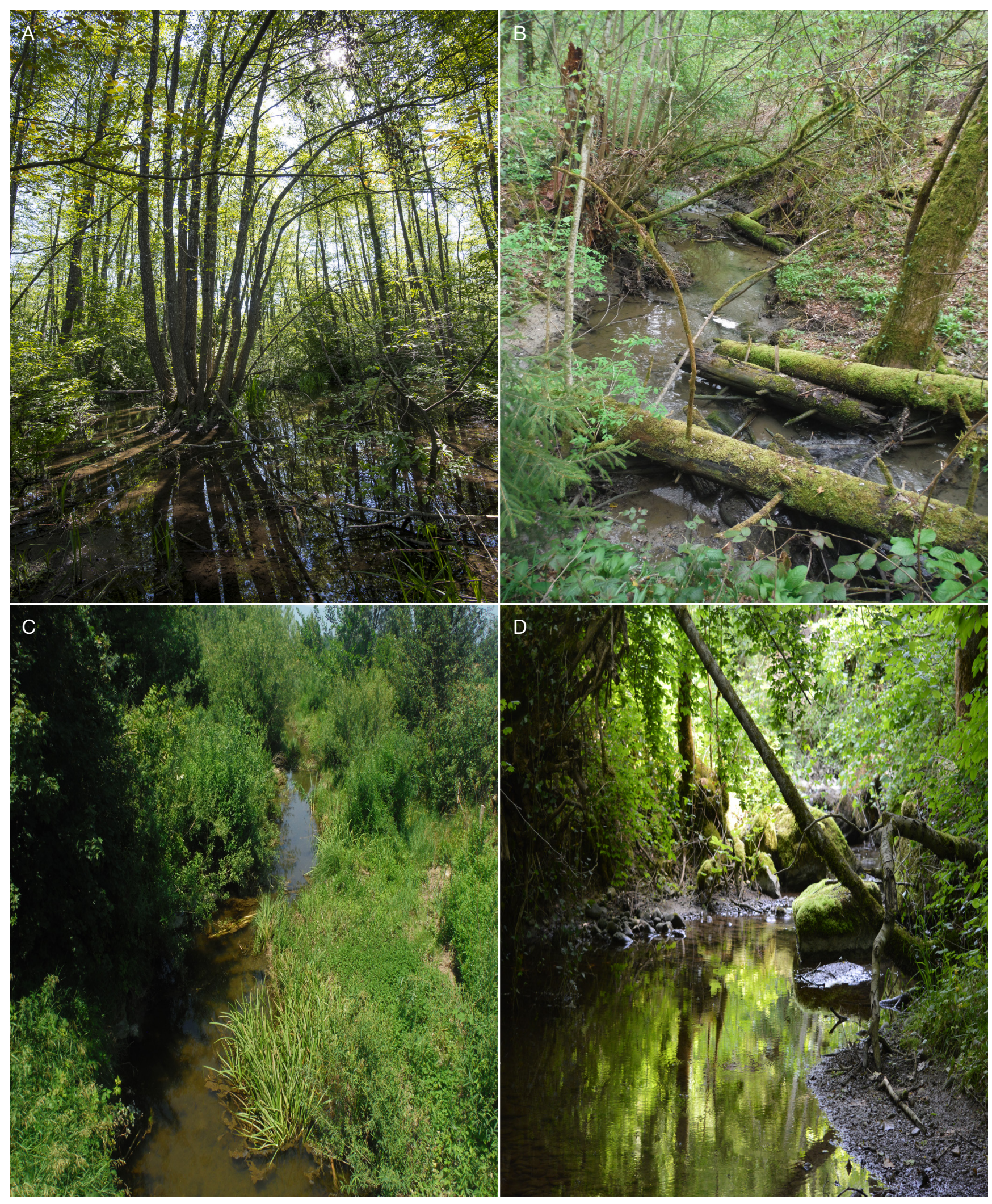

FIG. 2. - Illustration de la diversité des ripisylves prises en compte dans cette étude pour tester l'influence du score de l'Indice de Biodiversité et de Connectivité des Ripisylves et de chacun de ses facteurs constitutifs sur la richesse et la composition des communautés d'oiseaux. Crédits photos: S. De Danieli/INRAE (A) \& C. Gilles/FNE (B, C, D). 
TABLEAU 2. - Présentation des trois modèles les plus performants, parmi 17 modèles correspondant à l'Indice de Biodiversité et de Connectivité des Ripisylves, à chacun des 15 facteurs et au modèle nul, utilisés pour prédire les variations de l'Indice ponctuel d'Abondance (IPA) et de la richesse en oiseaux des milieux forestiers, agricoles, aquatiques et bâtis/rocheux le long des ripisylves, évalués par le critère d'information d'Akaike (AICc). La valeur de l'AICc, la différence d'AICc entre le meilleur modèle et les modèles successifs ( $\Delta$ AICc), le poids d'Akaike $(\mathbf{W})$ et le coefficient de détermination $\left(\mathbf{R}^{2}\right)$ sont présentés dans le tableau.

\begin{tabular}{|c|c|c|c|c|c|}
\hline Variable & Modèle & AlCc & $\triangle \mathrm{AlCc}$ & $\mathbf{W}$ & $\mathbf{R}^{2}$ \\
\hline Note IPA & $\begin{array}{l}M \\
C \\
N\end{array}$ & $\begin{array}{l}399,1 \\
402,6 \\
403,1\end{array}$ & $\begin{array}{l}0,000 \\
3,549 \\
3,976\end{array}$ & $\begin{array}{l}0,576 \\
0,098 \\
0,079\end{array}$ & $\begin{array}{l}0,148 \\
0,088 \\
0,081\end{array}$ \\
\hline $\begin{array}{l}\text { Espèces des milieux } \\
\text { forestiers }\end{array}$ & $\begin{array}{c}\text { IBCR } \\
\text { Nul } \\
\mathrm{N}\end{array}$ & $\begin{array}{l}223,8 \\
226,1 \\
226,2\end{array}$ & $\begin{array}{l}0,000 \\
2,254 \\
2,360\end{array}$ & $\begin{array}{l}0,262 \\
0,085 \\
0,081\end{array}$ & $\begin{array}{l}0,081 \\
0,000 \\
0,039\end{array}$ \\
\hline $\begin{array}{l}\text { Espèces des milieux } \\
\text { agricoles }\end{array}$ & $\begin{array}{l}M \\
E \\
G\end{array}$ & $\begin{array}{l}266,4 \\
274,6 \\
274,9\end{array}$ & $\begin{array}{l}0,000 \\
8,108 \\
8,405\end{array}$ & $\begin{array}{l}0,914 \\
0,016 \\
0,014\end{array}$ & $\begin{array}{l}0,204 \\
0,072 \\
0,066\end{array}$ \\
\hline $\begin{array}{l}\text { Espèces des milieux } \\
\text { aquatiques }\end{array}$ & $\begin{array}{c}\mathrm{O} \\
\mathrm{M} \\
\mathrm{I}\end{array}$ & $\begin{array}{l}182,7 \\
183,8 \\
184,9\end{array}$ & $\begin{array}{l}0,000 \\
1,160 \\
2,278\end{array}$ & $\begin{array}{l}0,300 \\
0,168 \\
0,096\end{array}$ & $\begin{array}{l}0,118 \\
0,098 \\
0,078\end{array}$ \\
\hline $\begin{array}{l}\text { Espèces des milieux } \\
\text { bâtis/rocheux }\end{array}$ & $\begin{array}{c}\mathrm{G} \\
\mathrm{M} \\
\mathrm{IBCR}\end{array}$ & $\begin{array}{l}185,0 \\
185,3 \\
188,6\end{array}$ & $\begin{array}{l}0,000 \\
0,319 \\
3,587\end{array}$ & $\begin{array}{l}0,372 \\
0,317 \\
0,062\end{array}$ & $\begin{array}{l}0,142 \\
0,137 \\
0,080\end{array}$ \\
\hline
\end{tabular}

valeur de la note IPA aux variables explicatives et un modèle de distribution de poisson (GLM) pour relier la richesse en espèces aux variables explicatives. Nous avons ensuite classé les 17 modèles du plus performant au moins performant, en utilisant le critère d'information d'Akaike corrigé pour les faibles effectifs (AICc) (Burnham \& Anderson 2002) et avons extrait le sens de variation et la significativité des paramètres de chaque modèle individuel.

Dans un second temps, les analyses ont porté sur les variations de composition en considérant les espèces d'oiseaux présentes dans un minimum de $10 \%$ et un maximum de $90 \%$ des ripisylves étudiées ( $\mathrm{n}$ espèces $=41$ ). Pour déterminer comment le score moyen de l'IBCR ainsi que le score moyen des facteurs individuels influencent la composition en espèces, nous avons utilisé des analyses canoniques en coordonnées principales (CAP, Anderson \& Willis 2003), basées sur une matrice des distances de Jaccard, avec 999 permutations. Compte tenu du fait que les facteurs individuels combinés donnent la note IBCR, c'est-à-dire de l'absence d'indépendance entre ces valeurs, nous avons réalisé deux CAPs, la première testant l'effet de l'IBCR seul sur la composition, la seconde testant l'effet conjoint des 15 facteurs. Nous avons ensuite calculé la contribution marginale des facteurs à l'inertie totale contrainte, c'est-à-dire la part de la variation expliquée par chacun des facteurs, et testé la signification statistique de ces contributions individuelles.

Dans un troisième temps, les analyses ont porté sur la réponse de chaque espèce d'oiseaux présente dans un minimum de $10 \%$ et un maximum de $90 \%$ des ripisylves étudiées $(n=41)$. Nous avons utilisé des modèles logistiques (distribution binomiale) pour déterminer comment la présence/absence de chacune de ces espèce était influencée, d'une part, par le score moyen de l'IBCR, d'autre part, par le score moyen de chacun des 15 facteurs constitutifs de l'indice. Pour identifier les facteurs qui permettent le mieux d'expliquer la probabilité de présence des espèces, nous avons hiérarchisé les 15 modèles, plus le modèle nul, via l'AICc. Nous avons ensuite considéré qu'un facteur influençait fortement une espèce lorsque la différence d'AICc entre le modèle de premier rang et les modèles successifs était supérieure à $2(\triangle \mathrm{AICc}>2)$ et le poids d'Akaike supérieur à 0,5 .

\section{RÉSULTATS}

Au total, 91 espèces d'oiseaux ont été inventoriées sur les 53 ripisylves étudiées (moyenne \pm écart type $=20,9 \pm 5,1$ ) . L'Indice ponctuel d'Abondance (IPA) varie entre 13,5 et 59,5 $(36,6 \pm 10,7)$; la richesse en oiseaux des milieux forestiers varie entre 3 et $11(7,3 \pm 1,7)$; la richesse en oiseaux des milieux agricoles varie entre 4 et $18(10,2 \pm 3,3)$; la richesse en oiseaux des milieux aquatiques varie entre 0 et $7(1,7 \pm 1,6)$; la richesse en oiseaux des milieux bâtis/rocheux varie entre 0 et $5(1,6 \pm 1,6)$.

Concernant les variations de diversité, nos résultats montrent que les valeurs de la note IPA sont mieux prédites par la connectivité longitudinale (M) (Tableau 2), comparativement aux autres facteurs, avec un effet significatif négatif (Tableau 3). L'IPA diminue aussi significativement avec l'augmentation des abris racinaires aquatiques $(G)$ et augmente significativement avec la quantité de bois mort sur pied (C) et la connectivité transversale (N) (Tableau 3). Pour les oiseaux des milieux forestiers, le score moyen de l'IBCR (Fig. 3), comparativement aux autres facteurs, permet de mieux prédire la richesse avec un effet significatif positif. Pour les oiseaux des milieux agricoles, la connectivité longitudinale (M), comparativement aux autres facteurs, permet de mieux prédire la richesse avec un effet significatif négatif. Pour les oiseaux des milieux aquatiques, la connectivité paysagère $(\mathrm{O})$, comparativement aux autres facteurs, permet de mieux prédire la richesse avec un effet significatif négatif. La richesse en espèces des milieux aquatiques diminue aussi significativement avec une augmentation de la connectivité longitudinale $(\mathrm{M})$ et de la présence de milieux aquatiques complémentaires au cours d'eau (I). Enfin, pour les oiseaux des milieux bâtis/rocheux, la présence d'abris racinaires 
TABLEAU 3. - Sens de variation et significativité (en gras, intervalle de confiance au seuil de $95 \%$ exclu 0) du coefficient de régression pour l'Indice de Biodiversité et de Connectivité des Ripisylves et les facteurs individuels utilisées pour prédire les variations de la note d'Indice ponctuel d'Abondance (IPA) et de la richesse en oiseaux des milieux forestiers, agricoles, aquatiques et bâtis/rocheux dans les ripisylves.

\begin{tabular}{|c|c|c|c|c|c|}
\hline \multirow[b]{2}{*}{ Facteurs } & \multicolumn{5}{|c|}{ Valeurs estimées ( \pm erreur type) } \\
\hline & IPA & Forestières & Agricoles & Aquatiques & Bâtis/rocheux \\
\hline IBCR & $-0,012( \pm 0,201)$ & $0,014( \pm 0,007)$ & $-0,006( \pm 0,006)$ & $-0,026( \pm 0,015)$ & $-0,031( \pm 0,015)$ \\
\hline$A$ & $1,049( \pm 1,084)$ & $0,016( \pm 0,037)$ & $0,017( \pm 0,032)$ & $0,117( \pm 0,075)$ & $0,081( \pm 0,077)$ \\
\hline $\mathrm{B}$ & $1,498( \pm 1,120)$ & $-0,009( \pm 0,039)$ & $0,045( \pm 0,033)$ & $0,110( \pm 0,082)$ & $0,085( \pm 0,083)$ \\
\hline $\mathrm{C}$ & $4,671( \pm 2,101)$ & $0,069( \pm 0,070)$ & $0,029( \pm 0,062)$ & $-0,071( \pm 0,167)$ & $0,007( \pm 0,156)$ \\
\hline $\mathrm{D}$ & $-0,770( \pm 1,134)$ & $0,042( \pm 0,037)$ & $-0,042( \pm 0,035)$ & $-0,175( \pm 0,101)$ & $-0,015( \pm 0,083)$ \\
\hline$E$ & $-0,734( \pm 1,177)$ & $0,051( \pm 0,038)$ & $-0,071( \pm 0,037)$ & $0,028( \pm 0,081)$ & $0,104( \pm 0,076)$ \\
\hline $\mathrm{F}$ & $0,868( \pm 1,214)$ & $0,022( \pm 0,042)$ & $0,045( \pm 0,036)$ & $0,062( \pm 0,089)$ & $-0,113( \pm 0,083)$ \\
\hline $\mathrm{G}$ & $-1,669( \pm 0,807)$ & $0,023( \pm 0,028)$ & $-0,047( \pm 0,025)$ & $-0,057( \pm 0,060)$ & $-0,177( \pm 0,065)$ \\
\hline $\mathrm{H}$ & $0,722( \pm 1,892)$ & $0,000( \pm 0,065)$ & $-0,024( \pm 0,057)$ & $0,053( \pm 0,122)$ & $0,184( \pm 0,098)$ \\
\hline I & $-0,668( \pm 1,105)$ & $0,025( \pm 0,037)$ & $-0,029( \pm 0,032)$ & $-0,167( \pm 0,084)$ & $-0,159( \pm 0,085)$ \\
\hline J & $-1,737( \pm 1,139)$ & $0,012( \pm 0,039)$ & $-0,035( \pm 0,035)$ & $-0,181( \pm 0,105)$ & $-0,053( \pm 0,090)$ \\
\hline $\mathrm{K}$ & $0,573( \pm 0,855)$ & $-0,016( \pm 0,029)$ & $0,041( \pm 0,025)$ & $0,082( \pm 0,063)$ & $0,059( \pm 0,063)$ \\
\hline $\mathrm{L}$ & $-0,491( \pm 1,122)$ & $0,031( \pm 0,037)$ & $-0,007( \pm 0,033)$ & $-0,062( \pm 0,084)$ & $-0,002( \pm 0,081)$ \\
\hline$M$ & $-4,619( \pm 1,555)$ & $0,068( \pm 0,059)$ & $-0,163( \pm 0,046)$ & $-0,257( \pm 0,109)$ & $-0,310( \pm 0,110)$ \\
\hline $\mathrm{N}$ & $3,559( \pm 1,679)$ & $0,084( \pm 0,058)$ & $0,046( \pm 0,050)$ & $0,001( \pm 0,123)$ & $-0,171( \pm 0,132)$ \\
\hline $\mathrm{O}$ & $0,595( \pm 1,738)$ & $0,052( \pm 0,059)$ & $-0,019( \pm 0,050)$ & $-0,310( \pm 0,123)$ & $-0,210( \pm 0,124)$ \\
\hline
\end{tabular}

aquatiques $(\mathrm{G})$, comparativement aux autres facteurs, permet de mieux prédire la richesse avec un effet significatif négatif. La richesse en espèces des milieux bâtis/rocheux diminue aussi significativement avec une augmentation du score moyen de l'IBCR et de la connectivité longitudinale (M).

Concernant la composition en espèces d'oiseaux, nos résultats montrent que l'IBCR seul explique 2,9\% $(\mathrm{p}=0,012)$ de la variation entre les ripisylves étudiées. Les 15 facteurs constitutifs de l'IBCR expliquent ensemble 33,2 \% ( $\mathrm{p}=0,001)$ de la variation de la composition entre les ripisylves (Fig. 4). Cette part de variation, c'est-à-dire l'inertie contrainte, est significativement expliquée par la connectivité longitudinale $(\mathrm{M})$, à hauteur de $9,3 \%(\mathrm{p}=0,001)$, les milieux minéraux et annexes $(\mathrm{J})(8,5 \%, \mathrm{p}=0,016)$, les abris racinaires aquatiques $(\mathrm{G})(8,3 \%, \mathrm{p}=0,037)$, les très gros bois vivants $(\mathrm{E})$ $(8,1 \%, p=0,022)$, les milieux aquatiques complémentaires au cours d'eau (I) $(7,3 \%, p=0,012)$, les dégradations et perturbations du milieu (L) $(6,9 \%, p=0,004)$, la connectivité paysagère $(\mathrm{O})(5,8 \%, \mathrm{p}=0,024)$, le bois mort au sol (D) $(5,8 \%, p=0,014)$ et la structure verticale de la végétation $(B)$ $(5,5 \%, 0,039)$. Les autres facteurs (A, C, F, H, K, N), bien qu'expliquant collectivement $34,4 \%$ de l'inertie contrainte, n'influencent pas de manière significative la composition en espèces d'oiseaux dans les ripisylves étudiées.

Concernant la réponse individuelle des espèces d'oiseaux à l'IBCR, nos résultats montrent que 22 espèces sont négativement influencées par l'indice, dont cinq significativement (Corbeau freux, Hypolaïs polyglotte, Hirondelle rustique, Héron cendré et Chardonneret élégant), et que 19 espèces sont positivement influencées par l'indice, dont deux significativement (Roitelet triple-bandeau et Pouillot véloce) (Fig. 5). Concernant les facteurs individuels, 15 espèces sont très fortement reliées à l'un des 15 facteurs, c'est-à-dire que la différence d'AICc entre le modèle de premier rang et les modèles successifs est supérieure à 2 et le poids d'Akaike pour ce même modèle est supérieur à 0,5 (Fig. 4). Spécifiquement, six espèces sont significativement mieux prédites par la connectivité longitudinale (effet négatif: Étourneau sansonnet, Fauvette grisette, Héron cendré, Hirondelle rustique, Rossignol philomèle; effet positif: Milan noir); deux espèces sont significativement mieux prédites par le nombre de bois mort au sol (effet négatif: Hypolaïs polyglotte et Verdier d'Europe) et deux espèces sont significativement mieux prédites par le nombre de très gros bois vivant (effet positif: Grimpereau des jardins et Hirondelle de fenêtre). Enfin la Mésange à longue queue est significativement mieux prédite par la structure verticale de la végétation (effet positif), le Loriot d'Europe par la présence d'abris racinaires aquatiques (effet négatif), la Sittelle torchepot par la continuité temporelle de l'état boisé (effet négatif), la Bergeronnette des ruisseaux par la présence de milieux aquatiques complémentaires au cours d'eau (effet négatif) et la Grive musicienne par la présence d'espèces exotiques (effet négatif).

\section{DISCUSSION}

D’une manière générale, nos résultats montrent que les cortèges d'oiseaux répondent de façon assez cohérente aux valeurs de l'Indice de Biodiversité et de Connectivité des Ripisylves (IBCR). Cela s'illustre par le fait que la richesse en oiseaux des milieux forestiers augmente avec le score moyen de l'IBCR alors que la richesse en oiseaux des milieux agricoles, aquatiques et bâtis/rocheux tend à diminuer. L'IBCR étant avant tout un outil de diagnostic des capacités d'accueil des boisements riverains pour les espèces forestières, la cohérence des relations biologiques ici mises en évidence pointe l'intérêt de l'indice proposé. Plus généralement, les résultats trouvés s'accordent avec ceux obtenus pour l'Indice de Biodiversité Potentielle (IBP), pour lequel des liens empiriques entre facteurs et cortèges d'oiseaux forestiers ont été mis en évidence (par exemple avec une augmentation de la richesse en espèces avec le nombre d'essences, la densité de très gros arbres ou bien encore le nombre de gros bois mort au sol; Larrieu et al. 2019). 

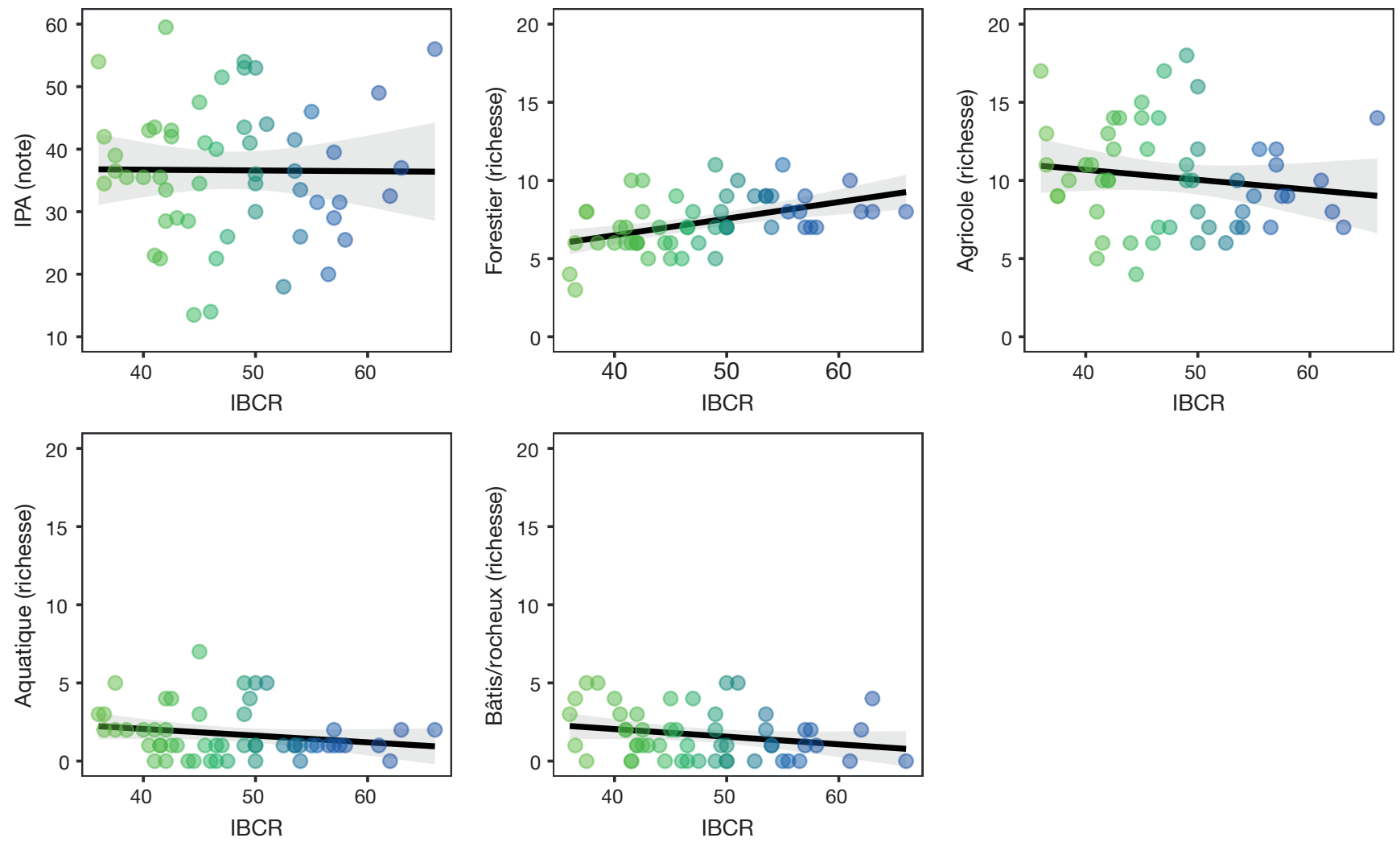

FIG. 3. - Variations des valeurs de l'Indice ponctuel d'Abondance (IPA) et de la richesse en oiseaux des milieux forestiers, agricoles, aquatiques et bâtis/rocheux dans les ripisylves, en fonction des valeurs de l'Indice de Biodiversité et de Connectivité des Ripisylves (IBCR).

\section{INFLUENCE DES FACTEURS DE L'IBCR}

\section{SUR LA RICHESSE EN ESPÈCES D'OISEAUX}

Concernant les variations de diversité, nos résultats montrent que seule la richesse en espèces des milieux forestiers augmente avec les valeurs de l'IBCR (Tableau 3; Fig. 3). Bien que la magnitude du changement mesuré ne soit pas très forte, ce résultat indique tout de même que la combinaison des 15 facteurs constitutifs de l'IBCR capture une part significative de la variabilité entre les ripisylves étudiées, en terme de qualité des peuplements forestiers. Aussi, bien que non significatif, il est intéressant de noter qu'à l'exception des facteurs relatifs à la structure verticale de la végétation et à la présence d'espèces exotiques qui ont un effet négatif, l'ensemble des facteurs décrivant l'état du peuplement, son contexte et sa connectivité, a un effet positif sur la richesse en oiseaux forestiers (Tableau 3). Appliqués aux ripisylves des petits et moyens cours d'eau permanents en contexte agricole, les résultats obtenus semblent donc suffisamment cohérents pour justifier de l'intérêt de l'outil développé pour quantifier la qualité des ripisylves pour la diversité des oiseaux forestiers. Inversement, nos résultats montrent que les variations des valeurs de l'IPA et de la richesse en espèces des milieux agricoles, aquatiques et bâtis/rocheux répondent globalement négativement aux valeurs de l'IBCR et aux différents facteurs le constituant. Ainsi, pour ces quatre variables à expliquer, l'augmentation de la connectivité longitudinale entraîne une diminution significative des valeurs de l'IPA et de la richesse en espèces. Cela montre que les ripisylves les moins connectées, c'est-à-dire ayant un couvert arboré discontinu et fragmenté dans l'espace, bénéficient avant tout aux communautés d'oiseaux utilisant les boisements de manière complémentaire aux habitats environnants non boisés. Spécifiquement, nos résultats montrent que ce sont avant tout les espèces agricoles et aquatiques qui utilisent ces fragments de ripisylve de moindre qualité, probablement comme zones de refuge complémentaires aux cultures/ pâtures pour les premières ou aux milieux aquatiques/ humides adjacentes pour les secondes. Plus généralement, cela montre que comparativement aux facteurs décrivant la qualité du peuplement de la ripisylve, les facteurs décrivant la connectivité spatiale des ripisylves sont importants à prendre en compte pour mieux comprendre la structuration des communautés d'oiseaux (Barbaro et al. 2007). Cela est particulièrement vrai pour des espèces très mobiles, ayant un grand domaine vital, comme la plupart des oiseaux, pour lesquelles assez peu de relations significatives ont été mises en évidence entre les facteurs de l'IBP et la richesse en espèces, comparativement à d'autres groupes taxonomiques comme les coléoptères saproxyliques, les bryophytes ou les syrphes (Larrieu et al. 2019). Pour mieux évaluer la biodiversité potentielle d'un peuplement forestier, il semble donc important de considérer quelques facteurs complémentaires, décrivant le contexte paysager dans lequel le peuplement se trouve, comme des mesures de connectivité proposées dans l'IBCR. L'utilisateur gardera cependant en mémoire que la prise en compte des facteurs de connectivité 


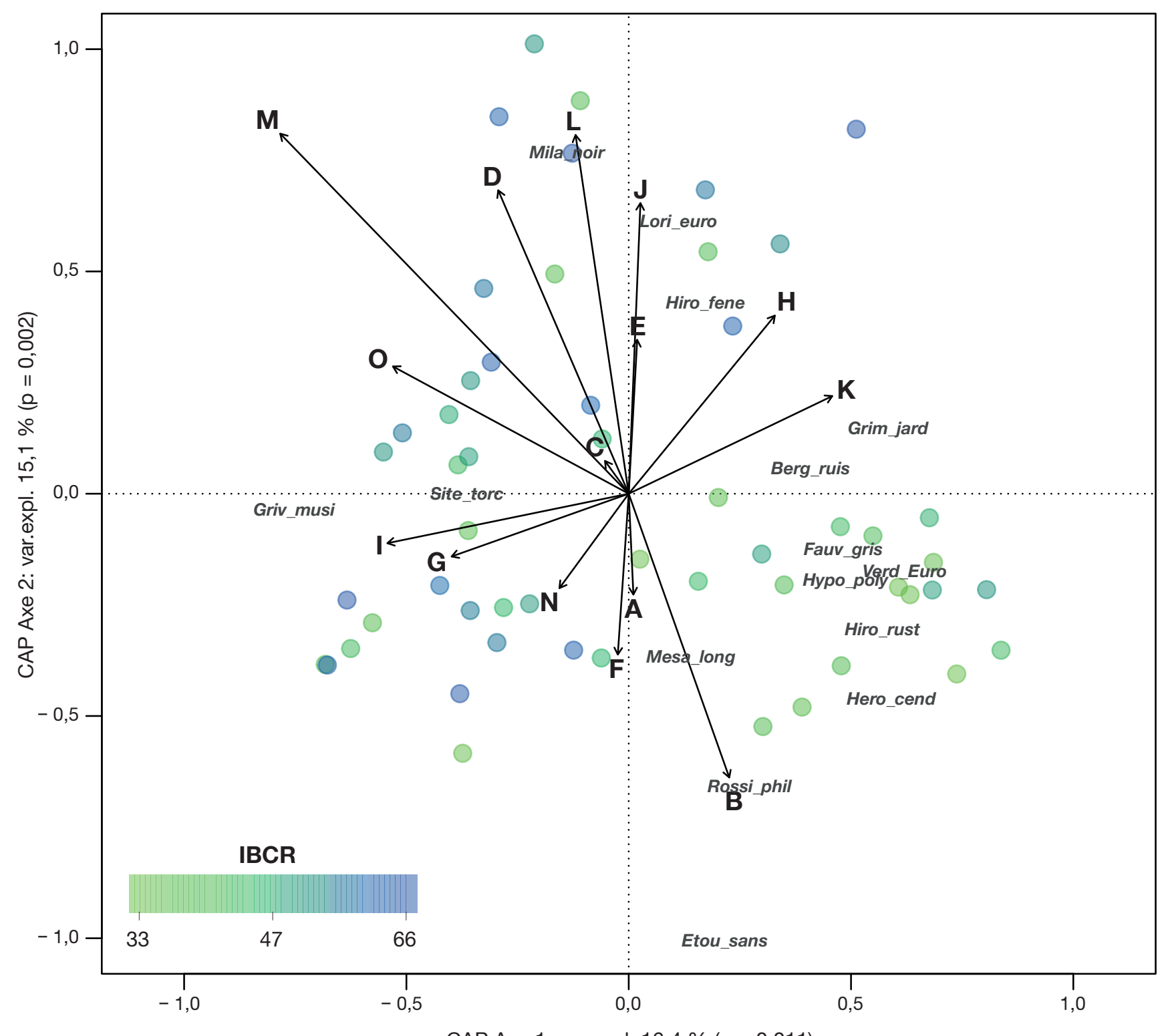

CAP Axe 1: var.expl. $16.4 \%(p=0.011)$

FIG. 4. - Analyse canonique en coordonnées principales illustrant l'effet des 15 facteurs constitutifs de l'IBCR sur la composition en oiseaux le long des ripisylves. Les espèces fortement influencées par un des facteurs, sur la base des analyses en présence/absence $(\Delta \mathrm{AICc}>2$ et poids d'Akaike $>0,5)$, sont également représentées : Berg_ruis = Bergeronnette des ruisseaux, Etou_sans = Étourneau sansonnet, Fauv_gris = Fauvette grisette, Grim_jard = Grimperau des jardins, Griv_musi $=$ Grive musicienne, Hero_cend $=$ Héron cendré, Hiro_fene $=$ Hirondelle de fenêtre, Hiro_rust $=$ Hirondelle rustique, Hypo_poly = Hypolaïs polyglotte, Lori_euro $=$ Loriot d'Europe, Mesa_long = Mésange à longue queue, Mila_noir = Milan noir, Rossi_phil = Rossignol philomèle, Site_torc $=$ Sittelle torchepot, Verd_Euro = Verdier d'Europe. Voir l'Annexe 1 pour les noms latins des espèces représentées. Abréviations: CAP, Analyse canonique en Coordonnées principales; IBCR, Indice de Biodiversité et de Connectivité des Ripisylves; $\mathbf{p}$, valeur de p; var.expl., variance expliquée.

demande des investigations complémentaires, à réaliser au bureau, et complexifiant dans une certaine mesure l'utilisation de l'outil. Finalement, nos résultats ont également mis en évidence des relations contre-intuitives, comme c'est le cas de la diminution de la richesse en oiseaux des milieux aquatiques avec le nombre de milieux aquatiques complémentaires au cours d'eau. Dans l'IBCR, la prise en compte de ces milieux est restreinte aux abords immédiats du cours d'eau et concerne, pour notre zone d'étude, des habitats de faible étendue sous couvert arboré (suintements, ruisselets, fossés humides, zones marécageuses). Aussi, dans la typologie des habitats que nous avons utilisée pour construire les guildes (Roché et al. 2016), le domaine "aquatique" rassemble des espèces nichant dans des milieux occupant a priori de vastes étendues - plans d'eau, roselières, marais, torrents, rivières à lit mobile et ripislyves - et, pour cinq d'entre eux, non liés directement aux boisements riverains. Il est donc possible que la réponse de ce groupe, très influencée par l'occurrence du Canard colvert et du Héron cendré, soit surtout sous la dépendance d'éléments paysagers non pris en compte par l'IBCR et inversement corrélés à l'espace occupé par la ripisylve et les milieux forestiers adjacents. 


\section{INFLUENCE DES FACTEURS DE L'IBCR}

\section{SUR LA COMPOSITION DES CORTĖGES D'OISEAUX}

Concernant la composition en espèces d'oiseaux, nos résultats montrent qu'une assez large proportion de la variation (33,6\%) est expliquée lorsque les différents facteurs de l'IBCR sont considérés conjointement. Neuf des 15 facteurs de l'IBCR ont un effet significatif sur les variations de composition entre les 53 ripisylves étudiées, soulignant ainsi la contribution individuelle de ces différents facteurs pour décrire efficacement la qualité des ripisylves pour les oiseaux. Aussi, comme pour les variations de richesse spécifique, les composantes du paysage environnant les ripisylves ont un effet fort sur la composition des communautés d'oiseaux (Barbaro et al. 2007). En effet, parmi les 15 facteurs constitutifs de l'IBCR, le facteur décrivant la connectivité longitudinale des ripisylves explique la plus forte proportion de variation de composition. Sans entrer dans le détail des contributions individuelles des différents facteurs pour les communautés d'oiseaux, leur bonne distribution dans les deux axes du plan factoriel de l'analyse canonique en coordonnées principales (Fig. 4) suggère la complémentarité des 15 facteurs entre eux. Il est cependant important de noter que l'absence de significativité de l'effet de certains facteurs vis-à-vis des oiseaux n'implique pas leur absence d'influence sur la biodiversité des ripisylves. En effet, l'influence de facteurs environnementaux sur la biodiversité mesurée dépend:

- du modèle d'étude, avec par exemple des exigences d'habitats très différentes entre oiseaux, coléoptères saproxyliques et plantes vasculaires;

- de la méthode employée pour échantillonner les taxons;

- du degré de finesse dans la caractérisation des attributs des peuplements, avec par exemple pour le bois mort la prise en compte des stades de décomposition;

- de l'étendue des variations que peut prendre chaque facteur individuel.

Ainsi, dans notre étude, l'absence d'effet des ligneux autochtones caractéristiques peut simplement traduire le fait que le dispositif a été construit sans échantillonner des ripisylves très dégradées, dominées par exemple par des plantations de peupliers gérées intensivement (Archaux \& Martin 2009).

\section{INFLUENCE DES FACTEURS DE L'IBCR}

\section{SUR LA RÉPONSE INDIVIDUELLE DES OISEAUX}

Au-delà de la réponse globale des communautés en termes de diversité et de composition, nos résultats à l'échelle des espèces prises individuellement viennent conforter l'idée que les ripisylves dégradées et déconnectées favorisent surtout la présence d'espèces d'oiseaux des milieux agricoles. Ainsi, bien que peu d'espèces parmi les 41 testées répondent significativement à l'IBCR, il est intéressant de voir que les espèces agricoles répondent globalement négativement à l'indice alors que les espèces forestières répondent globalement positivement (Fig. 5). Spécifiquement, plusieurs espèces non forestières (Étourneau sansonnet, Fauvette grisette, Héron cendré, Hirondelle rustique, Hypolaïs polyglotte, Mésange à longue queue, Rossignol philomèle, Verdier d'Europe) forment un groupe relativement cohérent dans l'espace factoriel de l'analyse

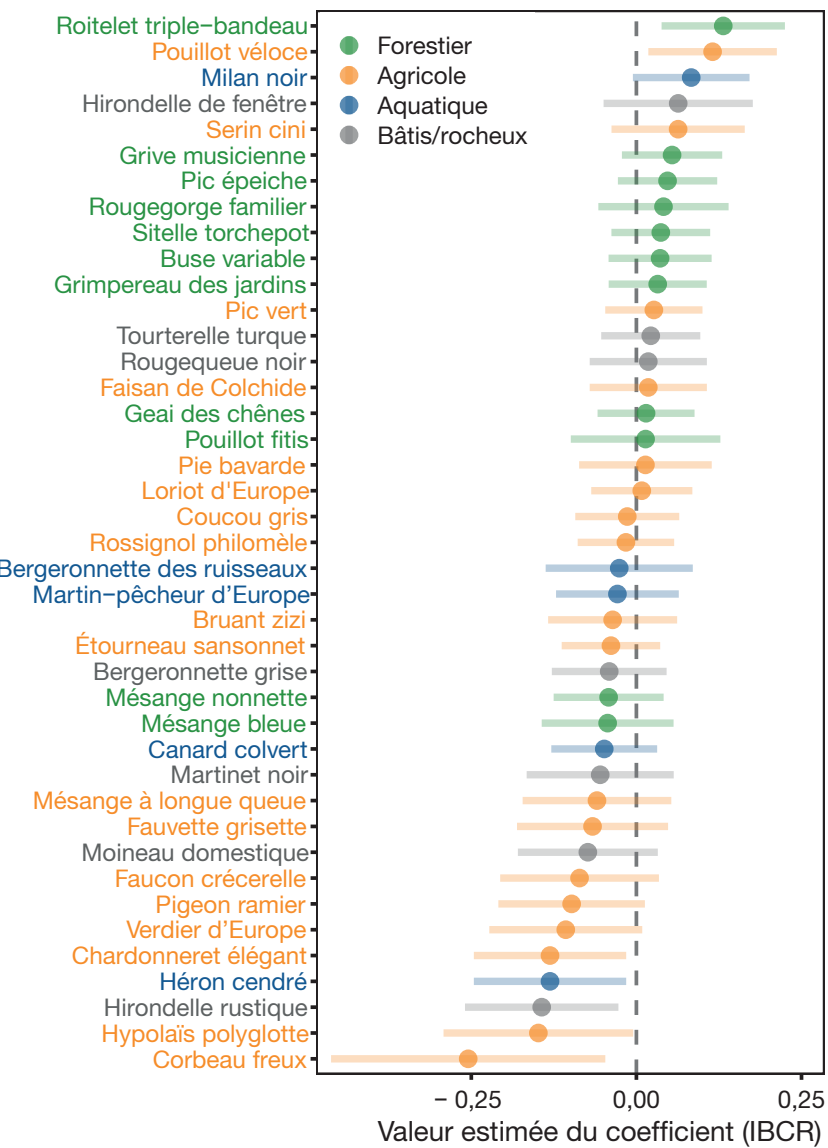

FIG. 5. - Réponse individuelle de 41 espèces d'oiseaux, présentes respectivement dans plus de $10 \%$ et moins de $90 \%$ des ripisylves étudiées, à l'Indice de Biodiversité et de Connectivité des Ripisylves (IBCR) (pour chaque espèce, le point représente la valeur estimée du coefficient de régression et la barre l'intervalle de confiance au seuil de $95 \%$ ). Voir l'Annexe 1 pour les noms latins des espèces représentées.

canonique en coordonnées principales, qui est très étroitement lié à la présence de ripisylves ayant une valeur d'IBCR faible, c'est-à-dire de faible qualité (Fig. 4). Inversement, les espèces forestières (Grimpereau des jardins, Grive musicienne, Sittelle torchepot) ou de ripisylves (Loriot d'Europe et Milan noir) sont réparties de manière hétérogène dans l'espace factoriel, soulignant que les exigences d'habitat au sein de cette guilde sont plus hétérogènes et donc que la qualité des ripisylves pour ces espèces dépend de facteurs variés, parfois opposés. En effet, la présence de certaines espèces spécialistes au sein des ripisylves peut dépendre de plusieurs composantes qui, si elles ne sont pas conjointement disponibles, peuvent compromettre les capacités d'établissement. Par exemple, la présence de la Sittelle torchepot est conditionnée par la disponibilité d'anciennes loges de pic et de bois de haute futaie. Aussi, certaines relations négatives avec des espèces $a$ priori associées aux ripisylves, comme c'est le cas du Héron cendré avec l'IBCR et la connectivité longitudinale, peuvent s'expliquer par les choix faits lors de la construction du plan d'échantillonnage. En effet, cette étude fait un focus délibéré sur les petits cours d'eau, sur les berges desquels le développement d'une ripisylve dense et continue va favoriser une fermeture du milieu peu 
propice aux hérons arboricoles, utilisant les arbres comme perchoirs (Fig. 2). Appliqué au contexte des vallées alluviales plus larges, il est en revanche possible que cet effet s'inverse et que la présence du Héron cendré se corrèle positivement aux valeurs de l'IBCR. Par ailleurs, les choix méthodologiques faits pour inventorier les oiseaux peuvent aussi entrainer des biais d'interprétation. La méthode des IPA repose en effet sur un protocole très généraliste, qui favorise la détectabilité des espèces qui s'entendent bien et se voient facilement, au détriment des espèces discrètes, souvent forestières (Bouvreuil pivoine, Pouillot fitis, Roitelet triple-bandeau). Dans ce cadre, il est possible que les inventaires soient incomplets mais aussi que certaines espèces soient absentes d'un habitat a priori favorable car elles n'ont pas eu le temps de le coloniser, compte tenu de contrainte à la dispersion agissant à plus large échelle. Enfin, il est tout simplement possible que certaines espèces soient sous la dépendance de facteurs environnementaux non pris en compte par l'IBCR et répondent donc de manière indirecte aux 15 facteurs retenus.

\section{CONCLUSIONS}

Tout comme l'IBP (Larrieu \& Gonin 2008), l'Indice de Biodiversité et de Connectivité des Ripisylves doit être vu comme un outil de diagnostic et d'aide à la gestion pour évaluer rapidement les principales caractéristiques des boisements riverains. Il ne donnera pas une mesure précise de qualité ou de biodiversité, mais il fournira aux gestionnaires des cours d'eau des éléments concrets pour orienter les choix de gestion, de conservation et de restauration de ces linéaires boisés. En renseignant de nombreux facteurs potentiellement très structurants pour la biodiversité, l'IBCR permet de dresser un diagnostic rapide quant à la capacité d'accueil des ripisylves. L'intérêt de cet outil est ici illustré avec des données de biodiversité réelle, limitées cependant aux seules communautés d'oiseaux. Comme cela a été fait pour l'IBP (Larrieu et al. 2019), il serait intéressant d'étudier la réponse d'autres groupes taxonomiques, ayant des exigences d'habitat et des capacités de dispersion contrastées, pour mieux appréhender l'influence relative des 15 facteurs de l'IBCR. Aussi, de même que pour l'IBP (Larrieu et al. 2019), des relations non attendues entre mesures de biodiversité réelle et certains facteurs ont été mises en évidence. Cela traduit un effet imprévu de certaines composantes de l'environnement jugées a priori négativement (par exemple certaines essences exotiques fournissent des ressources complémentaires pour les espèces) ou bien l'effet sous-jacent d'un autre facteur environnemental non mesuré mais corrélé avec le facteur de l'IBCR. Dans ce cadre et dans le prolongement de cette étude, il serait intéressant, d'une part, de tester la pertinence de cet outil dans une plus large gamme de conditions écologiques, en incluant notamment des ripisylves très dégradées, d'autre part, de tester la sensibilité des facteurs en dehors du domaine d'application défini (bassin de la Seine ou de la Loire) et au niveau des ripisylves des grands cours d'eau du bassin RhôneMéditerranée. Finalement, il convient de mentionner que l'utilisation de cet outil ne peut se substituer à des inventaires taxonomiques ciblés et complémentaires visant à évaluer la biodiversité réelle des ripisylves, notamment lorsque les gestionnaires sont confrontés à des enjeux de conservation forts, comme par exemple la conservation d'espèces patrimoniales.

\section{Remerciements}

Cette étude a bénéficié du support financier de l'Agence de l'Eau Rhône-Méditerrané Corse, de la Compagnie nationale du Rhône, de la DRAAF, de la Région Auvergne-RhôneAlpes et de la DREAL Auvergne-Rhône-Alpes dans le cadre du projet "Préserver et restaurer les ripisylves: un enjeu de biodiversité» (https://www.fne-aura.org/ripisylves, dernière consultation le 10 avril 2020). Nous tenons également à remercier Jacques Comolet-Tirman et Stanislas Wroza qui ont enrichi le manuscrit par leur relecture.

\section{RÉFÉRENCES}

Anderson M. J. \& Willis T. J. 2003. - Canonical analysis of principal coordinates: a useful method of constrained ordination for ecology. Ecology 84: 511-525. https://doi.org/10.1890/00129658(2003)084[0511:CAOPCA]2.0.CO;2

ARCHAUX F. \& MARTIN H. 2009. - Hybrid poplar plantations in a floodplain have balanced impacts on farmland and woodland birds. Forest Ecology and Management 257: 1474-1479. https:// doi.org/10.1016/j.foreco.2008.12.021

Barbaro L., Rossi J.-P., Vetillard F., Nezan J. \& Jactel H. 2007. - The spatial distribution of birds and carabid beetles in pine plantation forests: the role of landscape composition and structure. Journal of Biogeography 34: 652-664. https://doi. org/10.1111/j.1365-2699.2006.01656.x

Blondel J., Frochot B. \& Ferry C. 1970. — La méthode des Indices Ponctuels d'Abondance (1.P.A.) ou des relevés d'avifaune par «stations d'écoute». Alauda 38: 55-71.

Burnham K. P. \& ANDERSON D. R. 2002. - Model Selection and Multi-Model Inference: a Practical Information-Theoretic Approach. Springer-Verlag $2^{\text {nd }}$ edition, New York, 488 p.

DudGeOn D., ARThington A. H., Gessner M. O., KAWABATA Z.-I., Knowler D. J., Lévêque C., Naiman R. J., Prieur-Richard A.-H., Soto D., Stiassny M. L. J. \& Sullivan C. A. 2006. - Freshwater biodiversity: importance, threats, status and conservation challenges. Biological Reviews 81: 163-182. https:// doi.org/10.1017/s1464793105006950

DUFOUR S. \& PIÉGAY H. 2006. — Forêts riveraines des cours d'eau et ripisylves: spécificités, fonctions et gestion. Revue Forestière Française LVIII: 339-350. https://doi.org/10.4267/2042/6704

Dufour S., Barsoum N., Muller E. \& Piegay H. 2007. — Effects of channel confinement on pioneer woody vegetation structure, composition and diversity along the River Drôme (SE France). Earth Surface Processes and Landforms 32: 1244-1256. https:// doi.org/10.1002/esp.1556Gao T., Nielsen A. B. \& Hedblom M. 2015. - Reviewing the strength of evidence of biodiversity indicators for forest ecosystems in Europe. Ecological Indicators 57: 420-434. https://doi.org/10.1016/j.ecolind.2015.05.028

Gonzàlez Del Tànago M. \& García de Jalón D. 2011. Riparian Quality Index (RQI): a methodology for characterising and assessing the environmental conditions of riparian zones. Limnetica 30: 235-254. https://doi.org/10.23818/limn.30.18

Gregory S. V., Swanson F. J., Mckee W. A. \& Cummins K. W. 1991. - An ecosystem perspective of riparian zones. BioScience 41: 540-551. https://doi.org/10.2307/1311607 
Janssen P., Stella J., Piegay H., Rapple B., Pont B., Faton J.-M., Cornelissen J. H. C. \& EvetTe A. 2020. - Divergence of riparian forest composition and functional traits from natural succession along a degraded river with multiple stressor legacies. Science of the Total Environment 721: 137730. https://doi. org/10.1016/j.scitotenv.2020.137730

LARRIEU L. \& GONIN P. 2008. — L'indice de biodiversité potentielle (IBP): une méthode simple et rapide pour évaluer la biodiversité potentielle des peuplements forestiers. Revue Forestière Française LX: 727-748. https://doi.org/10.4267/2042/28373

Larrieu L., Gonin P. \& DeCONChat M. 2012. — Le domaine d'application de l'indice de biodiversité potentielle (IBP). Revue Forestière Française LXIV: 701-710. https://doi. org/10.4267/2042/50657

Larrieu L., Gosselin F., Archaux F., Chevalier R., Corriol G., Dauffy-Richard E., Deconchat M., Gosselin M., Ladet S., Savoie J.-M., Tillon L. \& Bouget C. 2018. — Cost-efficiency of cross-taxon surrogates in temperate forests. Ecological Indicators 87: 56-65. https://doi.org/10.1016/j.ecolind.2017.12.044

Larrieu L., Gosselin F., Archaux F., Chevalier R., CorRiol G., Dauffy-Richard E., Deconchat M., Gosselin M., Ladet S., Savoie J.-M., Tillon L. \& Bouget C. 2019. Assessing the potential of routine stand variables from multitaxon data as habitat surrogates in European temperate forests. Ecological Indicators 104: 116-126. https://doi.org/10.1016/j. ecolind.2019.04.085

MeIXLER M. S. \& BAIN M. B. 2010. — Landscape scale assessment of stream channel and riparian habitat restoration needs. Landscape and Ecological Engineering 6: 235-245. https://doi.org/10.1007/ s11355-010-0103-6

Munné A., Prat N., Sola C., Bonada N. \& Rieradevall M. 2003. - A simple field method for assessing the ecological quality of riparian habitat in rivers and streams: QBR index. Aquatic Conservation: Marine and Freshwater Ecosystems 13: 147-163. https://doi.org/10.1002/aqc.529

NAIMAN R. J. \& DeCAMPS H. 1997. - The ecology of interfaces: riparian zones. Annual review of Ecology and Systematics 28: 621658. https://doi.org/10.1146/annurev.ecolsys.28.1.621
Nilsson C., Reidy C. A., Dynesius M. \& Revenga C. 2005. Fragmentation and flow regulation of the world's large river systems. Science 308: 405-408. https://doi.org/10.1126/science. 1107887

Oswalt S. N. \& King S. L. 2005. - Channelization and floodplain forests: Impacts of accelerated sedimentation and valley plug formation on floodplain forests of the Middle Fork Forked Deer River, Tennessee, USA. Forest Ecology and Management 215: 69-83. https://doi.org/10.1016/j.foreco.2005.05.004

Perry L. G., Andersen D. C., Reynolds L. V., Nelson S. M. \& Shafroth P. B. 2012. - Vulnerability of riparian ecosystems to elevated $\mathrm{CO} 2$ and climate change in arid and semiarid western North America. Global Change Biology 18: 821-842. https://doi. org/10.1111/j.1365-2486.2011.02588.x

Poff N. L., Olden J. D., Merritt D. M. \& Pepin D. M. 2007. Homogenization of regional river dynamics by dams and global biodiversity implications. Proceedings of the National Academy of Sciences 104: 5732-5737. https://doi.org/10.1073/pnas.0609812104

QuANTUM GIS DeVELOPMENT TeAm 2016. — Quantum GIS Geographic Information System. Open Source Geospatial Foundation Project. http://qgis.osgeo.org

Roché J. E., Witté I., Comolet-Tirman J., Siblet J.-P., Cochet G., Deceuninck B., Frochot B., Guillot G., Muller Y., Nicolau-Guillaumet P. \& Olioso G. 2016. - Proposition de classification par l'habitat des oiseaux nicheurs de France: test de l'influence du niveau typologique sur des diagnostics de tendances. Alauda 84: 111-144.

Stokland J. N., Sittonen J. \& Jonsson B. G. 2012. — Biodiversity in Dead Wood. Cambridge University Press, Cambridge, 524 p. https://doi.org/10.1017/CBO9781139025843

Tonkin J. D., Merritt D. M., Olden J. D., Reynolds L. V. \& LYTLE D. A. 2018. - Flow regime alteration degrades ecological networks in riparian ecosystems. Nature Ecology \& Evolution 2: 86-93. https://doi.org/10.1038/s41559-017-0379-0

Weissteiner C., Ickerott M., Ott H., Probeck M., Ramminger G., Clerici N., Dufourmont H. \& De Sousa A. 2016. - Europe's green arteries - A continental dataset of riparian zones. Remote Sensing 8: 925. https://doi.org/10.3390/rs8110925 
ANNEXE

ANNEXE 1. - Liste des espèces d'oiseaux inventoriées dans les ripisylves des piémonts des Alpes du Nord et du Jura. Pour chaque espèce, le nom vernaculaire, le nom latin et le code utilisé dans les figures sont fournis.

\begin{tabular}{|c|c|c|}
\hline Nom vernaculaire & Nom latin & Code espèce \\
\hline Accenteur mouchet & Prunella modularis (Linnaeus, 1758) & Acce_mouc \\
\hline Alouette des champs & Alauda arvensis Linnaeus, 1758 & Alou_cham \\
\hline Alouette lulu & Lullula arborea (Linnaeus, 1758) & Alou_lulu \\
\hline Bergeronnette des ruisseaux & Motacilla cinerea Tunstall, 1771 & Berg_ruis \\
\hline Bergeronnette grise & Motacilla alba Linnaeus, 1758 & Berg_gris \\
\hline Bergeronnette printanière & Motacilla flava Linnaeus, 1758 & Berg_prin \\
\hline Bihoreau gris & Nycticorax nycticorax (Linnaeus, 1758) & Biho_gris \\
\hline Bouscarle de Cetti & Cettia cetti (Temminck, 1820) & Bous_cett \\
\hline Bruant jaune & Emberiza citrinella Linnaeus, 1758 & Brua_jaun \\
\hline Bruant zizi & Emberiza cirlus Linnaeus, 1766 & Brua_zizi \\
\hline Busard des roseaux & Circus aeruginosus (Linnaeus, 1758) & Busa_rose \\
\hline Buse variable & Buteo buteo (Linnaeus, 1758) & Buse_vari \\
\hline Canard colvert & Anas platyrhynchos Linnaeus, 1758 & Cana_colv \\
\hline Chardonneret élégant & Carduelis carduelis Linnaeus, 1758 & Char_eleg \\
\hline Choucas des tours & Corvus monedula (Linnaeus, 1758) & Chou_tour \\
\hline Chouette hulotte & Strix aluco Linnaeus, 1758 & Chou_hulo \\
\hline Cincle plongeur & Cinclus cinclus (Linnaeus, 1758) & Cinc_plon \\
\hline Corbeau freux & Corvus frugilegus Linnaeus, 1758 & Corb_freu \\
\hline Corneille noire & Corvus corone Linnaeus, 1758 & Corn_noir \\
\hline Coucou gris & Cuculus canorus Linnaeus, 1758 & Couc_gris \\
\hline Épervier d'Europe & Accipiter nisus (Linnaeus, 1758) & Eper_euro \\
\hline Étourneau sansonnet & Sturnus vulgaris Linnaeus, 1758 & Etou_sans \\
\hline Faisan de Colchide & Phasianus colchicus Linnaeus, 1758 & Fais_colc \\
\hline Faucon crécerelle & Falco tinnunculus Linnaeus, 1758 & Fauc_crec \\
\hline Faucon kobez & Falco vespertinus Linnaeus, 1766 & Fauc_kobe \\
\hline Faucon pèlerin & Falco peregrinus Tunstall, 1771 & Fauc_pele \\
\hline Fauvette à tête noire & Sylvia atricapilla (Linnaeus, 1758) & Fauv_noir \\
\hline Fauvette des jardins & Sylvia borin (Boddaert, 1783) & Fauv_jard \\
\hline Fauvette grisette & Sylvia communis Latham, 1787 & Fauv_gris \\
\hline Foulque macroule & Fulica atra Linnaeus, 1758 & Foul_macr \\
\hline Gallinule poule d'eau & Gallinula chloropus (Linnaeus, 1758) & Gall_poul \\
\hline Geai des chênes & Garrulus glandarius (Linnaeus, 1758) & Geai_chen \\
\hline Gobemouche gris & Muscicapa striata (Pallas, 1764) & Gobe_gris \\
\hline Goéland leucophée & Larus michahellis Naumann, 1840 & Goel_leuc \\
\hline Grand corbeau & Corvus corax Linnaeus, 1758 & Gran_Corb \\
\hline Grand cormoran & Phalacrocorax carbo (Linnaeus, 1758) & Gran_Corm \\
\hline Grande aigrette & Ardea alba Linnaeus, 1758 & Gran_Aigr \\
\hline Grèbe castagneux & Tachybaptus ruficollis Pallas, 1764 & Greb_cast \\
\hline Grimpereau des jardins & Certhia brachydactyla C.L. Brehm, 1820 & Grim_jard \\
\hline Grive draine & Turdus viscivorus Linnaeus, 1758 & Griv_drai \\
\hline Grive musicienne & Turdus philomelos C.L. Brehm, 1831 & Griv_musi \\
\hline Grosbec casse-noyau & Coccothraustes coccothraustes (Linnaeus, 1758) & Gros_cass \\
\hline Harle bièvre & Mergus merganser Linnaeus, 1758 & Harl_biev \\
\hline Héron cendré & Ardea cinerea Linnaeus, 1758 & Hero_cend \\
\hline Héron garde-bœufs & Bubulcus ibis (Linnaeus, 1758) & Hero_gard \\
\hline Hirondelle de fenêtre & Delichon urbicum (Linnaeus, 1758) & Hiro_fene \\
\hline Hirondelle rustique & Hirundo rustica Linnaeus, 1758 & Hiro_rust \\
\hline Huppe fasciée & Upupa epops Linnaeus, 1758 & Hupp_fasc \\
\hline Hypolaïs polyglotte & Hippolais polyglotta (Vieillot, 1817) & Hypo_poly \\
\hline Linotte mélodieuse & Linaria cannabina (Linnaeus, 1758) & Lino_melo \\
\hline Locustelle tachetée & Locustella naevia (Boddaert, 1783) & Locu_tach \\
\hline Loriot d'Europe & Oriolus oriolus (Linnaeus, 1758) & Lori_euro \\
\hline Martinet noir & Apus apus (Linnaeus, 1758) & Mart_noir \\
\hline Martin-pêcheur d'Europe & Alcedo atthis (Linnaeus, 1758) & Mart_pech \\
\hline Merle à plastron & Turdus torquatus Linnaeus, 1758 & Merl_plas \\
\hline Merle noir & Turdus merula Linnaeus, 1758 & Merl_noir \\
\hline Mésange à longue queue & Aegithalos caudatus (Linnaeus, 1758) & Mesa_long \\
\hline Mésange bleue & Cyanistes caeruleus (Linnaeus, 1758) & Mesa_bleu \\
\hline Mésange charbonnière & Parus major Linnaeus, 1758 & Mesa_char \\
\hline Mésange nonnette & Poecile palustris (Linnaeus, 1758) & Mesa_nonn \\
\hline Milan noir & Milvus migrans (Boddaert, 1783) & Mila_noir \\
\hline Moineau domestique & Passer domesticus (Linnaeus, 1758) & Moin_dome \\
\hline Mouette rieuse & Chroicocephalus ridibundus (Linnaeus, 1766) & Moue_rieu \\
\hline Perdrix rouge & Alectoris rufa (Linnaeus, 1758) & Perd_roug \\
\hline Pic épeiche & Dendrocopos major (Linnaeus, 1758) & Pic_epei \\
\hline Pic épeichette & Dendrocopos minor (Linnaeus, 1758) & Pic_ette \\
\hline
\end{tabular}


ANNEXE 1. - Suite.

\begin{tabular}{lll}
\hline Nom vernaculaire & Nom latin & Code espèce \\
\hline Pic mar & Dendrocopos medius (Linnaeus, 1758) & Pic_mar \\
Pic noir & Dryocopus martius (Linnaeus, 1758) & Pi__noir \\
Pic vert & Picus viridis Linnaeus, 1758 & Pic_vert \\
Pie bavarde & Pica pica (Linnaeus, 1758) & Pie_bava \\
Pie-grièche écorcheur & Lanius collurio Linnaeus, 1758 & Pieg_eco \\
Pigeon biset domestique & Columba livia J.F. Gmelin, 1789 & Pige_dome \\
Pigeon ramier & Columba palumbus Linnaeus, 1758 & Pige_rami \\
Pinson des arbres & Fringilla coelebs Linnaeus, 1758 & Pins_arbr \\
Pouillot fitis & Phylloscopus trochilus (Linnaeus, 1758) & Poui_fiti \\
Pouillot siffleur & Phylloscopus sibilatrix (Bechstein, 1793) & Poui_siff \\
Pouillot véloce & Phylloscopus collybita (Vieillot, 1817) & Poui_velo \\
Roitelet à triple bandeau & Regulus ignicapilla (Temminck, 1820) & Roit_trip \\
Rossignol philomèle & Luscinia megarhynchos C.L. Brehm, 1831 & Rossi_phil \\
Rougegorge familier & Erithacus rubecula (Linnaeus, 1758) & Roug_fami \\
Rougequeue à front blanc & Phoenicurus phoenicurus (Linnaeus, 1758) & Roug_frbc \\
Rougequeue noir & Phoenicurus Ochruros (S.G. Gmelin, 1774) & Roug_noir \\
Rousserolle effarvatte & Acrocephalus scirpaceus (Hermann, 1804) & Rous_effa \\
Serin cini & Serinus serinus (Linnaeus, 1766) & Seri_cini \\
Sitelle torchepot & Sitta europaea Linnaeus, 1758 & Site_torc \\
Tarier pâtre & Saxicola rubicola (Linnaeus, 1766) & Tari_pâtr \\
Torcol fourmilier & Jynx torquilla Linnaeus, 1758 & Torc_four \\
Tourterelle des bois & Streptopelia turtur (Linnaeus, 1758) & Tour_bois \\
Tourterelle turque & Streptopelia decaocto (Frivaldszky, 1838) & Tour_turq \\
Troglodyte mignon & Troglodytes troglodytes (Linnaeus, 1758) & Trog_mign \\
Verdier d'Europe & Chloris chloris (Linnaeus, 1758) & Verd_Euro \\
\hline
\end{tabular}

